\title{
Tip induced fluorescence quenching for nanometer optical and topographical resolution
}

\author{
Olaf Schulz 1,2,7, Zhao Zhao ${ }^{3,4}$, Alex Ward ${ }^{1,2}$, Marcelle Koenig ${ }^{5}$, Felix Koberling5 ${ }^{5}$, Yan Liü ${ }^{3,4}$, Jörg Enderlein ${ }^{6}$, \\ Hao $\mathrm{Yan}^{3,4}$ and Robert $\operatorname{Ros}^{1,2^{*}}$
}

\begin{abstract}
Progress in nanosciences and life sciences is closely related to developments of high resolution imaging techniques. We introduce a technique which produces correlated topography and fluorescence lifetime images with nanometer resolution. Spot sizes below $5 \mathrm{~nm}$ are achieved by quenching of the fluorescence with silicon probes of an atomic force microscope which is combined and synchronized with a confocal fluorescence lifetime microscope. Moreover, we demonstrate the ability to locate and resolve the position of two fluorescent molecules separated by $20.7 \mathrm{~nm}$ on a DNA origami triangle with $120 \mathrm{~nm}$ side length by correlating topography and fluorescence data. With this method, we anticipate applications in nano- and life sciences, such as the determination of the structure of macromolecular assemblies on surfaces, molecular interactions, as well as the structure and function of nanomaterials.
\end{abstract}

Keywords: Correlated atomic force and fluorescence microscopy, Optical ultraresolution microscopy, DNA nanostructures, Single molecule detection

\section{Introduction}

Nano- and life sciences progress in tandem with developments of high resolution imaging techniques. During the last decades, the invention and improvement of scanning probe microscopy techniques like atomic force microscopy (AFM) (Ando, 2012; Binnig et al., 1986; Engel \& Muller, 2000; Garcia \& Herruzo, 2012; Hinterdorfer et al., 2012) have offered new views onto nanoscale materials. Optical microscopy has been pushed beyond the diffraction limit and the observation of single molecules has evolved into a standard technology (Betzig et al., 2006; Dertinger et al., 2009; Dertinger et al., 2010b; Eggeling et al., 2009; Frey et al., 2006; Frey et al., 2004; Gustafsson, 2005; Hell, 2007; Hell \& Wichmann, 1994; Hillenbrand \& Keilmann, 2002; Moerner \& Fromm, 2003; Rust et al., 2006; van de Linde et al., 2012). Several approaches for simultaneous high resolution topographical and optical imaging have been realized (Harke et al., 2012; Hartschuh, 2008; Novotny, 2007; Noy \& Huser, 2003; Yang et al., 2000). Most of

\footnotetext{
* Correspondence: Robert.Ros@asu.edu

${ }^{1}$ Department of Physics, Arizona State University, Tempe AZ 85287, USA

${ }^{2}$ Center for Biological Physics, Arizona State University, Tempe AZ 85287, USA Full list of author information is available at the end of the article
}

these high resolution approaches exploit local field enhancement effects near sharp tips to increase the fluorescence emission of fluorophores in close proximity (Hartschuh, 2008). Optical resolutions below $10 \mathrm{~nm}$ are difficult to achieve. In a rare example, Quake et al. reported an optical resolution of $8 \mathrm{~nm}$ using tip induced fluorescence enhancement (Ma et al., 2006). However, enhancement of fluorescence depends strongly on the orientation of the molecule to the probe, which introduces uncertainty in the location and respective distance of molecules of different orientation. Xie and Dunn, and Ambrose et al. combined near field scanning optical microscopy (NSOM) and fluorescence lifetime imaging microscopy (FLIM) using metal coated near field probes to study the fluorescence emission of single dye molecules on surfaces with spatial resolutions in the range of about 100 - $300 \mathrm{~nm}$ (Ambrose et al., 1994; Xie \& Dunn, 1994). They observed a dependence of the fluorescence lifetime from the relative position of the near field probe, indicating quenching due to the metal coating.

In this work, we take advantage of localized fluorescence quenching of sharp silicon tips to create a novel high resolution imaging technique. We exploit this effect 
to spatially and temporally correlate optical and topographical imaging at sub $5 \mathrm{~nm}$ resolution. Standard, nonmodified AFM tips can be used for this tip induced quenching imaging (TIQI) ensuring high optical, as well as topographical resolutions. We present measurements with individual fluorophores and DNA origami (Rothemund, 2006) triangles with $120 \mathrm{~nm}$ sides into which two fluorophores are incorporated at $20.7 \mathrm{~nm}$ from each other. We were able to resolve the morphology of the DNA triangle and to confirm the localization and distance of the fluorophores.

The energy of an electronically excited fluorophore can either be used to emit a photon (radiation), or the energy can be transferred to another object or the environment (i.e. fluorophore, surface, solvent molecule, etc.) resulting in non-radiative decay. Several mechanisms are known to alter the fluorescence of a molecule by changing both the radiative and the non-radiative pathways. Quenching is the term used whenever this alteration results in detection of less fluorescence light. The process behind quenching can amongst others be explained by either dipole-dipole interaction, direct energy or electron transfer (Dexter, 1953; Forster, 1948). It has been shown that quenching can be utilized to probe distances in the nanometer or sub-nanometer range (Berndt et al., 2010; Marme et al., 2003; Selvin, 2000). The impact of metal tips or nanostructures close to fluorophores has been well characterized (Ambrose et al., 1994; Anger et al., 2006; Ebenstein et al., 2006; Eckel et al., 2007; Krug et al., 2005; Kuehn et al., 2006; Xie \& Dunn, 1994; Yoskovitz et al., 2011). Here, we exploit the quenching of organic fluorophores by sharp ndoped silicon tips to record topographical and optical images simultaneously with nanometer resolution.

\section{Methods}

The experimental setup consists of a MicroTime200 confocal fluorescence lifetime microscope (PicoQuant) with an inverted optical microscope (Olympus IX-71) onto which a sample scanning atomic force microscope (MFP-3D, Asylum Research) is mounted (Koenig et al., 2009). The setup, synchronization, and alignment have been described in detail (Schulz et al., 2010). Pulsed diode lasers were used for excitation at $640 \mathrm{~nm}$ and $470 \mathrm{~nm}$. The polarizations were linear along the slow scan axis (vertical in the images) for the single fluorophore images and circular for the DNA origamis. The circular polarization increased the chance to excite both fluorophores on one DNA origami equally. The alignment of the AFM probe with the confocal volume is realized by monitoring the laser light that is reflected back from the sample surface. Imaging the back reflected light creates a specific pattern, which is distorted by the AFM tip. For alignment, the objective is moved until the distortion is centered in this pattern. This procedure is quick, requires only low laser intensity (to reduce photo bleaching) and is reliably accurate within $10 \mathrm{~nm}$.

For the combined AFM/FLIM experiments with DNA origami triangles, a thin sheet of muscovite mica was deposited on a glass coverslide as described (Ma et al., 2006). These surfaces were incubated with $10 \mu \mathrm{l}$ of a solution of TAE buffer containing $10 \mu \mathrm{M} \mathrm{Mg}{ }^{2+}$ and $0.4 \mathrm{nM}$ DNA origami for $5 \mathrm{~min}$ and then successively rinsed with $200 \mu \mathrm{l}$ TAE buffer, $200 \mu \mathrm{l} \mathrm{MgCl}_{2}(200 \mathrm{mM})$ in water, and $400 \mu \mathrm{l}$ ethanol and then dried in a stream of nitrogen gas. The origami samples were prepared as described in (Rothemund, 2006) and Additional file 1. For the single dye experiments, a drop of solution containing the fluorophores was deposited on a glass cover slide to give a density of active fluorophores of about 1 molecule per $1 \mu \mathrm{m}^{2}$. The drop was allowed to dry on the surface, and the sample was used without further treatment. All combined experiments were performed under ambient conditions in contact mode.

\section{Results and discussions}

We utilize an AFM combined with a confocal fluorescence microscope with pulsed laser excitation (Figure 1a). The AFM collects information about the sample topography whereas the optical microscope records fluorescence intensity and fluorescence lifetime while scanning the sample. The alignment of the AFM probe and the microscope objective in combination with the synchronization of the two microscopes allows for the acquisition of temporally and spatially correlated topographical and fluorescence data (Schulz et al., 2010). In the combined measurements, the AFM tip, which is in direct contact with the sample surface, alters the optical response of the sample fluorophores. Figure $1 \mathrm{~b}$ shows a line scan over a single fluorophore on a glass surface excited with a wavelength of $640 \mathrm{~nm}$. The Gaussian shape of the curve reflects the diffraction limit of optical resolution in far field fluorescence microscopy. Additionally, a sharp dip that stems from the localized fluorescence quenching by the silicon AFM tip can be seen in the curve. The width of this dip and not the size of the diffraction-limited spot define the optical resolution. Figure 1c shows a fluorescence intensity image of single molecules on glass. The shapes of the spots reflect the shape and polarization of the excitation laser beam in the sample plane and the orientation of the molecule. Inside these spots, small quenching dips are visible. Figure 1d shows a smaller scan range of the same sample. Single dye molecules with quenching spots are clearly visible in this area. In Figure 1e the same area is scanned as in (d), but now with retracted AFM tip. The identical dye molecules not showing quenching dips are visible in this confocal fluorescence image. 


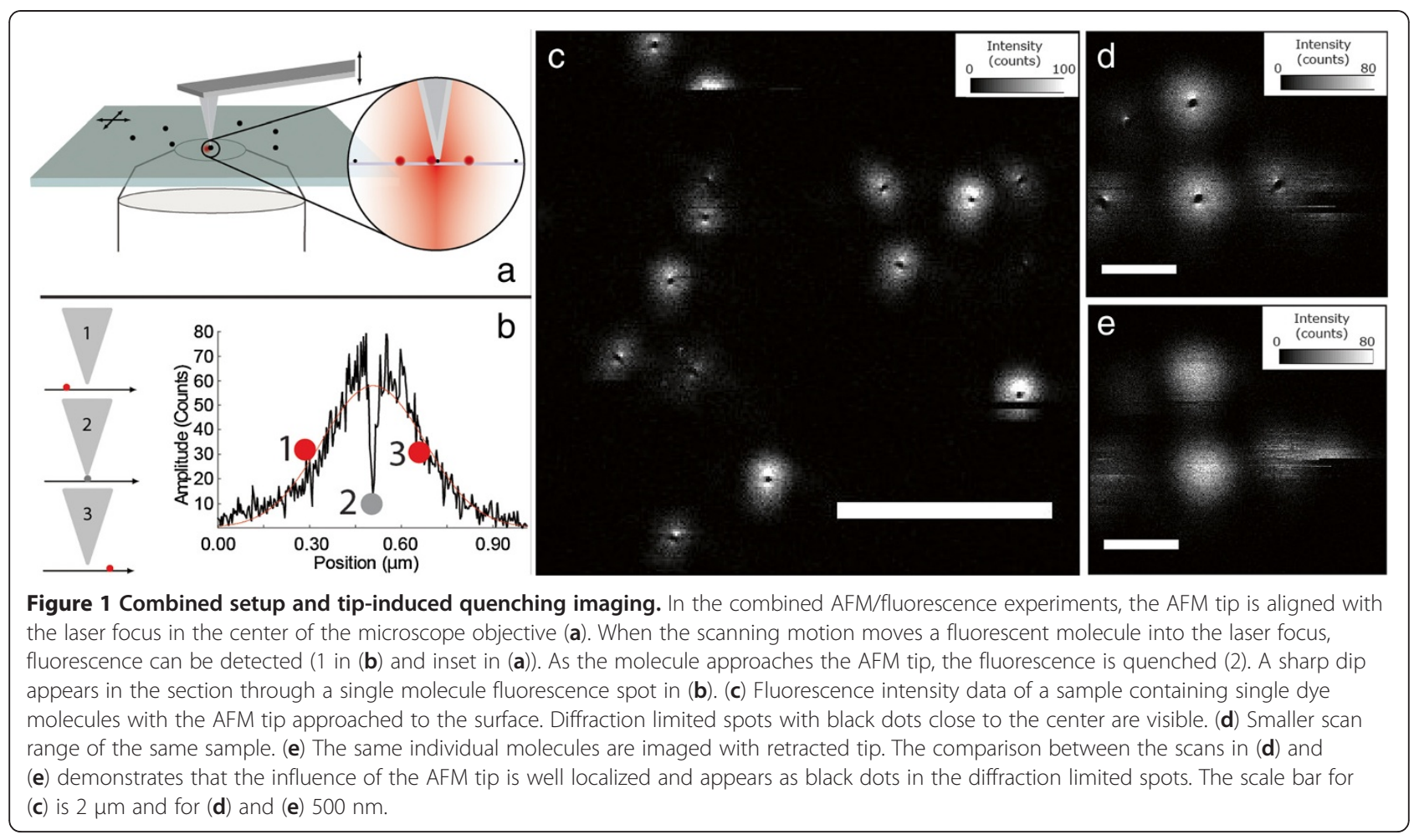

The width of the quenching dips and consequently the optical resolution depend strongly on the dimensions and shape of the AFM tip. For sharp tips, we see a distinct dark dot with a full width at half maximum (FWHM) less than $5 \mathrm{~nm}$ located inside the diffraction limited fluorescence spot (Figure 2a). The FWHM can be used as measure for the resolution (Dertinger et al., 2010a; Gerton et al., 2004; Huang et al., 2009; Ma et al., 2006; Westphal et al., 2003; Yoskovitz et al., 2010). Typical spot sizes are under $8 \mathrm{~nm}$ (Figure 2b-d). For blunter tips, the shape of the area of contact between the probe and the sample surface is reflected in the shape of the quenched dot (Figure 2e). The variation of the FWHM for different experiments with different AFM probes depends on many factors, including the care that is taken when approaching the probe to the surface.

High resolution scans reveal a quenching efficiency of up to $100 \%$ within background noise. Not only a change in the fluorescence intensity but also a change in fluorescence lifetime can be observed (Figure 2f). This result provides some physical insights into the effects that dominate the interaction between the fluorophore and the AFM tip. In the outer region of the dark spot, we find an area of shortened fluorescence lifetime which indicates that non-radiative energy transfer plays a role at very short distances from the tip (Aussenegg et al., 1987). The intensities and lifetimes at distances further from the tip are consistent with a radiative dipole induced dipole type feedback mechanism (Barnes, 1998) and geometrical considerations as outlined in Figure 2g. The fluorescence of a molecule near a surface depends strongly on the orientation of its dipole relative to the interface. The method of image dipoles can be used graphically to exploit the impact of this effect. From defocused imaging, (Marshall et al., 2011) we could show that the molecular transition dipole moments lie parallel to the sample surface (see Additional file 1). When the fluorophore is directly under the silicon tip and thus parallel to the tip surface, it creates an antiparallel oriented mirror dipole inside the tip. This creates an electric field that leads to negative feedback. In the case that the fluorophore is beside the tip, its dipole has a component normal to the silicon surface leading to positive feedback (Figure 2g). For very short distances also the vertical case results in quenching because of direct energy transfer to the silicon. The high localization of the quenched spot and the asymmetry in the data is related to the orientation between the tip and fluorophore, which changes swiftly as the molecule is scanned relative to the AFM tip (Figure 2g). We can evaluate this model by solving Maxwell's equations in planar structures in the presence of an oscillating electric dipole source (the emitting molecule) by using Sommerfeld integrals (Chizhik et al., 2009; Enderlein, 2000; Enderlein \& Ruckstuhl, 2005) and taking into account the change of the relative orientations of dipole and AFM tip surface during the scanning motion (Additional file 1). Comparison of our experimental data and the calculated fluorescence intensity images obtained 

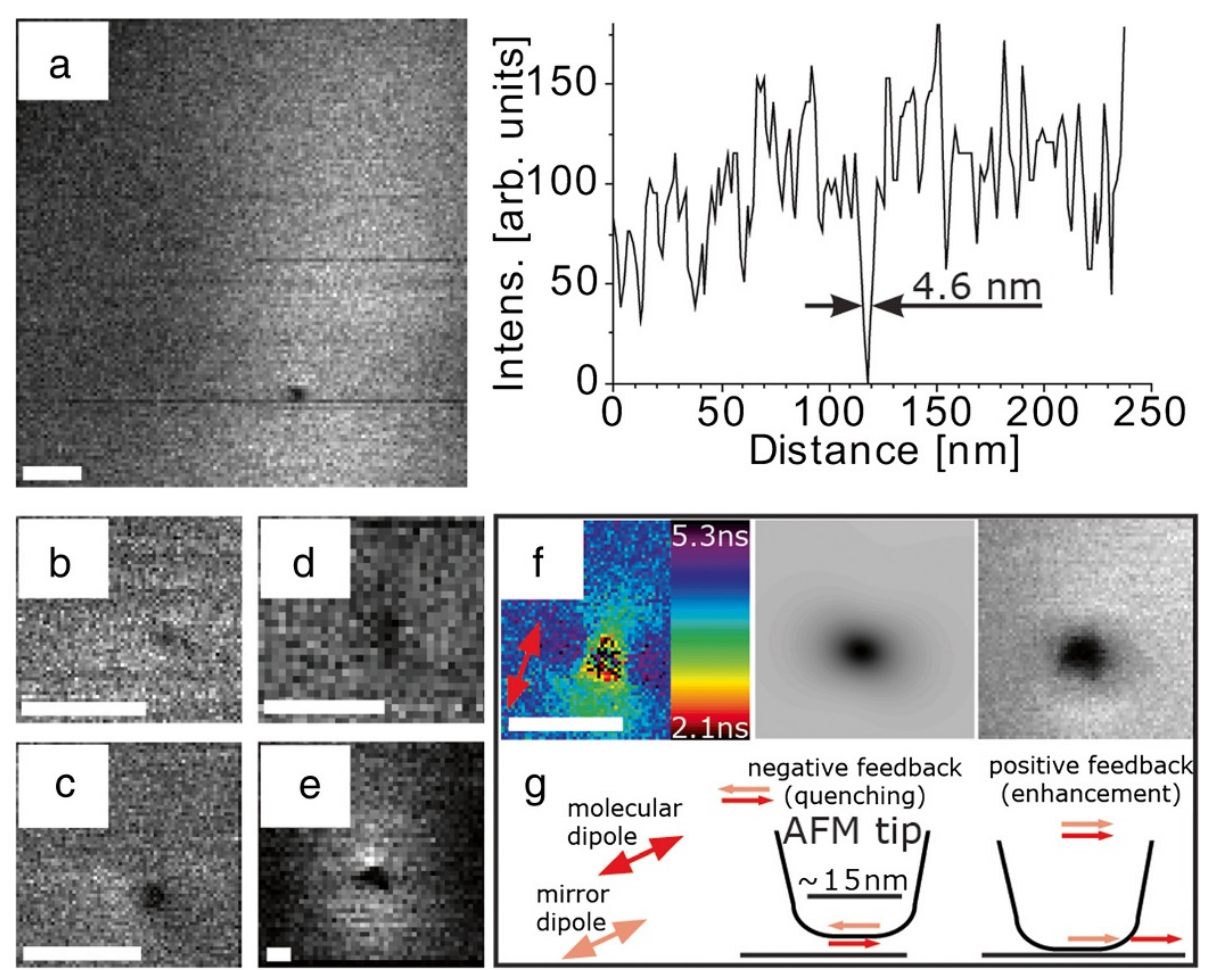

Figure 2 Resolution and mechanism of tip-induced quenching imaging. Quenching of single Atto655 molecules by the AFM tip is highly localized. For a sharp probe, the full width at half maximum (FWHM) of the quenched spot can be less than $5 \mathrm{~nm}(\mathbf{a})$. For (b), (c), (d) the FWHMs are $6.8 \mathrm{~nm}, 7.5 \mathrm{~nm}$, and $5.9 \mathrm{~nm}$, respectively. For blunt tips, the shape of the end of the tip can be clearly seen as a triangle (e). In (f) the fluorescence lifetime distribution, the calculated fluorescence intensity and the measured fluorescence intensity of a quenching spot with a FWHM of $16 \mathrm{~nm}$ is shown. The distribution of fluorescence intensities and lifetimes gives insight into the physical mechanisms that lead to quenching. The effect of the AFM tip on a fluorophore depends on the orientation and distance between them (g). For very small separations, parallel orientation leads to out-of-phase oscillation of the molecular dipole and its mirror image (g, middle). In the case of normal orientation (g, right), the dipole oscillates in phase. The scale bars in (a)-(f) are $50 \mathrm{~nm}$.

using this method show good agreement (Figure $2 \mathrm{f}$ and Additional file 1: Figure S4).

The interaction between the AFM probe and the fluorophore would be elucidated further by investigating the fluorescence response with changing tip-surface distance. In our experiments under ambient conditions, however, it was impossible to gather such data: strong capillary forces between the AFM probe and the surface dominate for short distances. Thus, when one approaches the AFM tip to the surface, a "snap-in" event can be observed; that means, at some distance, capillary forces start to dominate, and the AFM tip moves very quickly to the surface, within a time that cannot be resolved in the fluorescence data. When retracting the AFM tip from the surface, the opposite effect occurs, and the AFM probe is held on the surface until the restoring force of the cantilever exceeds the capillary forces. The length scale, at which the snap-in and snap-back occur, coincide with the length scale where the fluorescence is strongly modulated, such that no interesting information could be extracted from the force/fluorescence-distance curves that we acquired.
The quenching mechanism does not depend on the use of particular organic fluorophores. We observed fluorescence quenching with silicon tips for several different classes of dyes over a range of excitation and emission wavelengths. We tested successfully the oxazine derivative Atto655, as well as the cyanine Alexa647, and the rhodamine derivatives Atto647N and Alexa488 (Additional file 1: Figure S5).

Interestingly, for quantum dots embedded in $10 \mathrm{~nm}$ thin PMMA films, Ebenstein et al. did not observe quenching for bare silicon tips (Ebenstein et al., 2006). This may be due to the larger distance between the tip and the quantum dot and/or the thickness of the quantum dot shell.

To test the correlated optical and topographical resolution of our novel method, we used TIQI to resolve the position of single dye molecules on DNA nanostructures. For this application, we chose Atto655 because it was under our experimental conditions the most stable dye of the four that we used (Additional file 1). At a laser excitation intensity of $0.7 \mu \mathrm{W}$ in the focus, which resulted in a count rate of a few $\mathrm{kHz}$, we could image a single Atto655 
molecule's fluorescence over several minutes. Two Atto655 dye molecules were attached to the same edge of a DNA origami triangle with $120 \mathrm{~nm}$ sides. According to the DNA design the fluorophores should be separated by $20.7 \mathrm{~nm}$ (64 base pairs) (Figure 3a). A similar structure was used to compare super-resolution fluorescence microscopy techniques by Steinhauer et al. (Steinhauer et al., 2009). They were able to optically resolve two dye molecules separated by $89 \mathrm{~nm}$ on DNA rectangles. Figure 3b shows the fluorescence intensity of a single DNA triangle on mica and Figure 3c the corresponding AFM topography. The shape and position of the DNA nanostructure can be clearly seen in the AFM image. The quality of the AFM topography image is not as excellent as expected for highresolution AFM measurements. This is a result of mechanical noise which is coupled into the sample through the optical microscope, and it is a common problem for combining AFM with optical microscopy. For typical scan speeds of $2 \mu \mathrm{m} / \mathrm{s}$ and scan sizes $1 \mu \mathrm{m}^{2}$, we measure noise values between $0.3 \mathrm{~nm}$ and $0.6 \mathrm{~nm}$ (root mean square) for simultaneous AFM/FLIM measurements on mica samples compared to $0.1 \mathrm{~nm}$ for not combined AFM imaging. The use soft cantilevers allows us nondestructive imaging of the DNA origami samples in contact mode with a sufficient resolution to identify the position, size, shape, and orientation. For samples which are more prone to damage due to tip-sample interactions, our method can be used under liquid conditions, allowing a minimization of forces between the AFM tip and the sample surface.

The fluorescence intensity image in Figure 3b shows the overlay of the undistinguishable diffraction limited spots of the two dye molecules. A pattern that shows the outer-
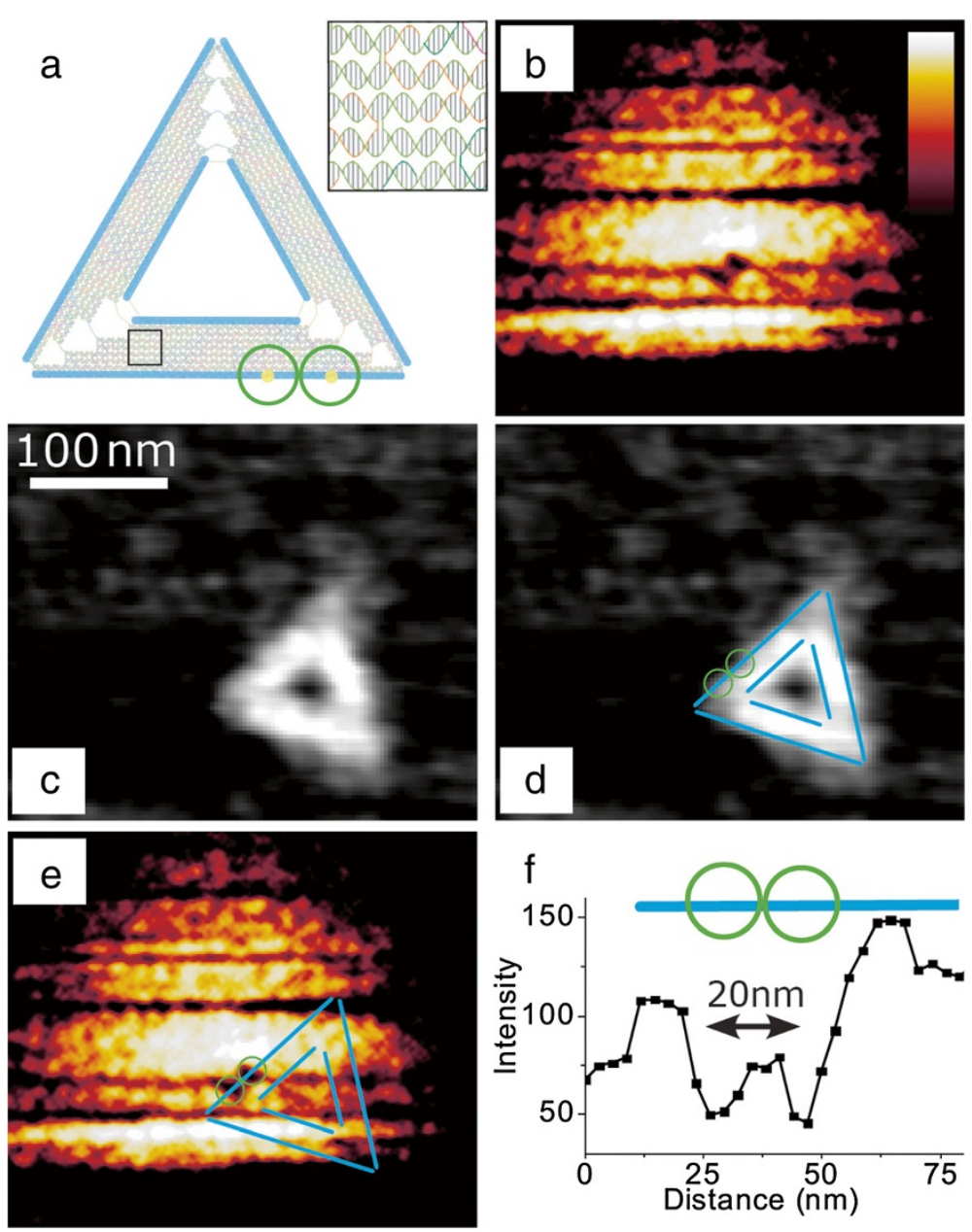

Figure 3 Combined topographic and fluorescence scans of DNA origami nanosctructures. DNA origami consists of many pieces of single stranded DNA that fold into an interlinked double helix structure (a). Two pieces of DNA are modified with the fluorescent dye Atto655 (yellow dots in (a)). Because of their unique position in the DNA nanostructure due to base pairing, they appear at the same position for every DNA triangle. The bottom side of the structure (blue line) is $120 \mathrm{~nm}$ long. The fluorescence intensity and the AFM topography are shown in (b) and (c), respectively. The position of the nanostructure is determined from the AFM image (d) and transferred to the fluorescence image (e) to confirm the position of the dyes with respect to the triangle geometry. (f) shows a section through the center of the dark spots from which their distance can be determined. The scale bar is the same for all panels. 
and innermost DNA strands in the structure and the position of the fluorophores on the DNA is shown superimposed on the AFM image in Figure 3d. This pattern is also shown superimposed on the fluorescence intensity image to compare the position of the quenched spots with the expected position determined from the topography image. Figure 3e shows that the quenched spots, within the experimental uncertainty, indeed coincide in position with the location of the fluorophores according to the topography image. The green circles in Figure 3 represent a $10 \mathrm{~nm}$ uncertainty based on the limitation of our optical and topographical resolution. Because of the overlapping diffraction limited spots, the fluorescence intensity in the dips falls to about $50 \%$ of the signal. It is worth noting that the topography image and the quenching image were not translated to match each other. This is possible because the two microscopes are synchronized, and the data is sorted into pixels according to the time at which it was acquired (Schulz et al., 2010). Since the tip records the topography at the molecule's position and changes its fluorescence at the same time, these features appear in the same position in both images.

Mica surfaces have a rather strong influence on the emission of the fluorophores; blinking and bleaching are much more prominent compared to the single dye molecules on glass. In fact, many of the DNA origamis that can be seen in the AFM images do not show fluorescence above background level. Also, even when both fluorophores of an origami are emitting during the scan, if one or both of them blink while the AFM probe is near the fluorophore, the quenching would be obscured. Another problem arises when DNA origamis with their fluorophores are too close to each other, which reduces the signal to noise ratio. Considering eight triangles, for which the two quenched spots could be clearly distinguished, we found an average distance between the fluorophores of $21 \mathrm{~nm} \pm 2 \mathrm{~nm}$ (s.d.) by measuring the distances between the two minima of the fluorescence intensities in sections like Figure 3f. This matches exceptionally well the expected value of $20.7 \mathrm{~nm}$ from the DNA design.

Figure 4 shows the overlaid fluorescence and AFM data for two different DNA origami triangles. By comparing the location of the quenched spots with the known structure, we can see that the triangles in a) and b) are mirrored. In essence this means that one of them is lying upside down on the surface. Since the fluorophores are attached to the edge of the DNA origami, the fluorophores are still readily accessible to the influence of the AFM tip. The figure also shows that the exact alignment of the tip with the confocal volume is not critical. Even for slightly misaligned scans like b) the quenching spots can still be observed. Figure 4b) also shows that bleaching of the molecules, which appears as a sudden reduction in fluorescence, does not affect TIQI, as long as it occurs after the AFM tip has scanned the molecule.

\section{Conclusions}

In this work, we have presented a novel method for simultaneous optical and topographical resolutions in the sub $5 \mathrm{~nm}$ range. This range bridges the atomic and the
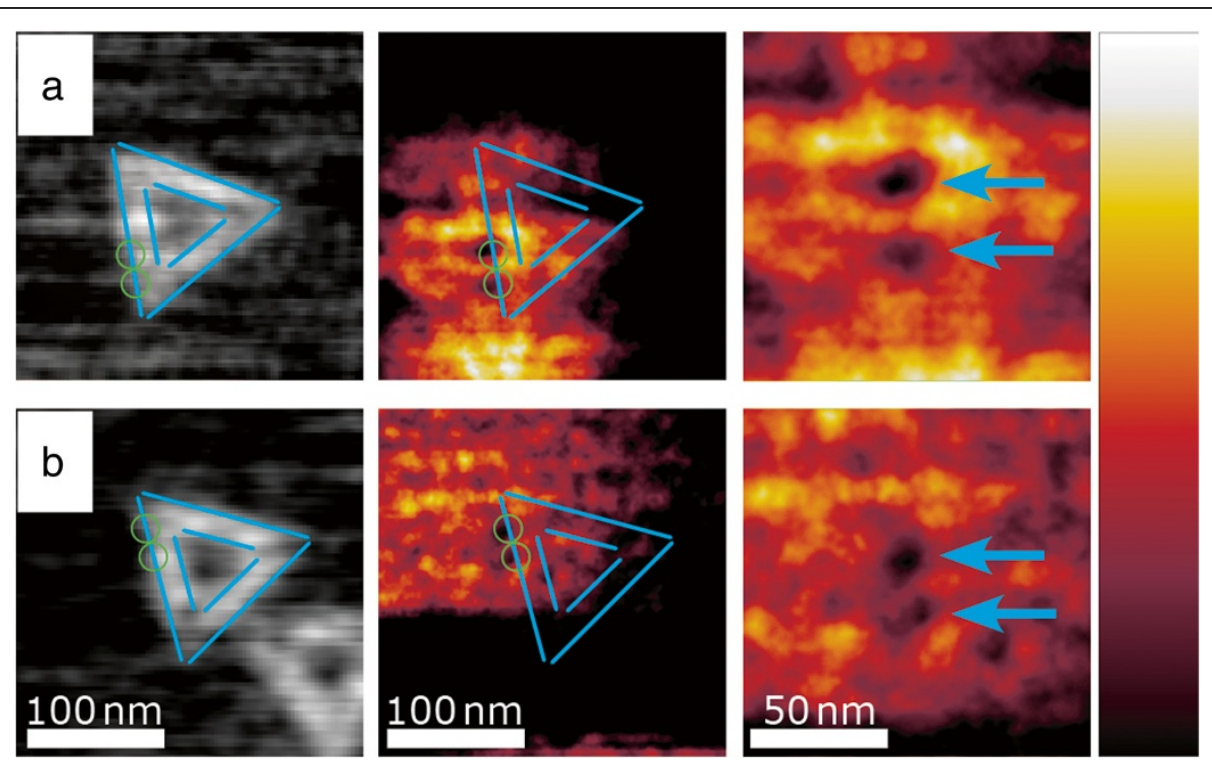

Figure 4 Fluorescence quenching in combined scans of DNA origami. Two examples of DNA origami scans in which quenching of both fluorophores is clearly visible. The comparison of the location of the quenched spots shows that the DNA structure in (a) is upside-down with respect to the one in (b). Thus, using quenching imaging on asymmetric structures, we can determine their orientation on the surface without ambiguity. The intensity scale in all images has been adjusted to give maximum visual contrast and the lowest value (black) is, in general, higher than zero counts. 
optical superresolution range, making it possible to address important questions in supramolecular chemistry, molecular biology, and nanosciences. The combination of topography and fluorescence adds high resolution chemical contrast to the high topographical resolution of AFM. This approach could be extended to multicolor imaging and to imaging under liquid conditions. The commercial availability of the instruments and the AFM tips should allow this method to spread rapidly into other fields.

\section{Additional file}

Additional file 1: The supporting information contains material about the orientation of the molecule on the sample surface, the modeling of the tip induced quenching, the application of different organic dye molecules and the preparation of the DNA origami nanostructures.

\section{Abbreviations}

AFM: Atomic force microscopy; FLIM: Fluorescence lifetime imaging microscopy; TIQI: Tip induced quenching imaging; FWHM: Full width at half maximum.

\section{Competing interests}

The authors declare that they have no competing interests.

\section{Authors' contributions}

OS and AW carried out the experiments; OS carried out the theoretical calculations and designed the geometrical model; ZZ prepared origami samples, MK and FK worked out and helped to implement the synchronization of the instruments; $Y L$ and $H Y$ designed the origami nanostructure research; JE designed the theoretical calculations; OS and RR analyzed the data; OS and RR wrote the paper; RR designed the research. All authors read and approved the final manuscript.

\section{Acknowledgements}

This work was supported by Arizona State University, ONR (N000140911118) and ASU Presidential Strategic Initiative Fund. The authors thank Dr. Anne K. Jones for proofreading the manuscript.

\section{Author details}

${ }^{1}$ Department of Physics, Arizona State University, Tempe AZ 85287, USA.

${ }^{2}$ Center for Biological Physics, Arizona State University, Tempe AZ 85287 USA. ${ }^{3}$ Biodesign Institute, Arizona State University, Tempe AZ 85287, USA ${ }^{4}$ Department of Chemistry and Biochemistry, Arizona State University, Tempe AZ 85287, USA. ${ }^{5}$ PicoQuant GmbH, Rudower Chaussee 29, Berlin 12489, Germany. ${ }^{6}$ III. Institute of Physics, Georg August University, Göttingen 37077 Germany. ${ }^{7}$ Present address: Ill. Institute of Physics, Georg August University, Göttingen 37077, Germany.

Received: 5 November 2012 Accepted: 24 January 2013 Published: 21 February 2013

\section{References}

Ambrose WP, Goodwin PM, Martin JC, Keller RA (1994) Alterations of singlemolecule fluorescence lifetimes in near-field optical microscopy. Science 265:364-367

Ando T (2012) High-speed atomic force microscopy coming of age. Nanotechnology 23:062001

Anger P, Bharadwaj P, Novotny L (2006) Enhancement and quenching of singlemolecule fluorescence. PhysRevLett 96:113002

Aussenegg FR, Leitner A, Lippitsch ME, Reinisch H, Riegler M (1987) Novel aspects of fluorescence lifetime for molecules positioned close to metal-surfaces. Surf Sci 189:935-945
Barnes WL (1998) Fluorescence near interfaces: the role of photonic mode density. J Mod Opt 45:661-699

Berndt M, Lorenz M, Enderlein J, Diez S (2010) Axial nanometer distances measured by fluorescence lifetime imaging microscopy. Nano Lett 10:1497-1500

Betzig E, Patterson GH, Sougrat R, Lindwasser OW, Olenych S, Bonifacino JS, Davidson MW, Lippincott-Schwartz J, Hess HF (2006) Imaging intracellular fluorescent proteins at nanometer resolution. Science 313:1642-1645

Binnig G, Quate CF, Gerber C (1986) Atomic force microscope. PhysRevLett 56:930-933

Chizhik A, Schleifenbaum F, Gutbrod R, Khoptyar D, Meixner AJ, Enderlein J (2009) Tuning the fluorescence emission spectra of a single molecule with a variable optical subwavelength metal microcavity. Phys Rev Lett 102:073002073006

Dertinger T, Colyer R, lyer G, Weiss S, Enderlein J (2009) Fast, background-free, 3D super-resolution optical fluctuation imaging (SOFI). P Natl Acad Sci USA 106:22287-22292

Dertinger T, Colyer R, Vogel R, Enderlein J, Weiss S (2010a) Achieving increased resolution and more pixels with superresolution optical fluctuation imaging (SOFI). Opt Express 18:18875-18885

Dertinger T, Heilemann M, Vogel R, Sauer M, Weiss S (2010b) Superresolution optical fluctuation imaging with organic dyes. Angew Chem Int Ed Engl 49:9441-9443

Dexter DL (1953) A theory of sensitized luminescence in solids. J Chem Phys 21:836-850

Ebenstein Y, Yoskovitz E, Costi R, Aharoni A, Banin U (2006) Interaction of scanning probes with semiconductor nanocrystals; physical mechanism and basis for near-field optical imaging. J Phys Chem A 110:8297-8303

Eckel R, Walhorn V, Pelargus C, Martini J, Enderlein J, Nann T, Anselmetti D, Ros R (2007) Fluorescence-emission control of single CdSe nanocrystals using goldmodified AFM tips. Small 3:44-49

Eggeling C, Ringemann C, Medda R, Schwarzmann G, Sandhoff K, Polyakova S, Belov VN, Hein B, von Middendorff C, Schoenle A, Hell SW (2009) Direct observation of the nanoscale dynamics of membrane lipids in a living cell. Nature 457:1159-1163

Enderlein J (2000) A theoretical investigation of single-molecule fluorescence detection on thin metallic layers. Biophys J 78:2151-2158

Enderlein J, Ruckstuhl T (2005) The efficiency of surface-plasmon coupled emission for sensitive fluorescence detection. Opt Express 13:8855-8865

Engel A, Muller DJ (2000) Observing single biomolecules at work with the atomic force microscope. NatStructBiol 7:715-718

Forster T (1948) Zwischenmolekulare Energiewanderung und Fluoreszenz. Ann Phys-Berlin 2:55-75

Frey HG, Witt S, Felderer K, Guckenberger R (2004) High-resolution imaging of single fluorescent molecules with the optical near-field of a metal tip. Phys Rev Lett 93:200801

Frey HG, Bolwien C, Brandenburg A, Ros R, Anselmetti D (2006) Optimized apertureless optical near-field probes with $15 \mathrm{~nm}$ optical resolution. Nanotechnology 17:3105-3110

Garcia R, Herruzo ET (2012) The emergence of multifrequency force microscopy Nat Nanotechnol 7:217-226

Gerton JM, Wade LA, Lessard GA, Ma Z, Quake SR (2004) Tip-enhanced fluorescence microscopy at 10 nanometer resolution. Phys Rev Lett 93:180801

Gustafsson MGL (2005) Nonlinear structured-illumination microscopy: wide-field fluorescence imaging with theoretically unlimited resolution. P Natl Acad SC USA 102:13081-13086

Harke B, Chacko JV, Haschke H, Canale C, Diaspro A (2012) A novel nanoscopic tool by combining AFM with STED microscopy. Optical Nanoscopy 1:3

Hartschuh A (2008) Tip-enhanced near-field optical microscopy. Angew Chem Int Ed Engl 47:8178-8191

Hell SW (2007) Far-field optical nanoscopy. Science 316:1153-1158

Hell SW, Wichmann J (1994) Breaking the diffraction resolution limit by stimulated-emission-depletion fluorescence microscopy. Opt Lett 19:780-782

Hillenbrand R, Keilmann F (2002) Material-specific mapping of metal/ semiconductor/dielectric nanosystems at $10 \mathrm{~nm}$ resolution by backscattering near-field optical microscopy. Appl Phys Lett 80:25-27

Hinterdorfer P, Garcia-Parajo MF, Dufrene YF (2012) Single-molecule imaging of cell surfaces using near-field nanoscopy. Acc Chem Res 45:327-336

Huang B, Bates M, Zhuang XW (2009) Super-resolution fluorescence microscopy. Annu Rev Biochem 78:993-1016 
Koenig M, Koberling F, Walters D, Viani J, Ros R, Schulz O (2009) Combining atomic force microscopy with confocal microscopy. http://www.picoquant. com/technotes/technote_mt200_afm.pdf; Accessed 19 Dec 2012

Krug JT, Sanchez EJ, Xie XS (2005) Fluorescence quenching in tip-enhanced nonlinear optical microscopy. Appl Phys Lett 86:233102

Kuehn S, Hakanson U, Rogobete L, Sandoghdar V (2006) Enhancement of singlemolecule fluorescence using a gold nanoparticle as an optical nanoantenna. Phys Rev Lett 97:017402

Ma ZY, Gerton JM, Wade LA, Quake SR (2006) Fluorescence near-field microscopy of DNA at sub-10 nm resolution. Phys Rev Lett 97:260801

Marme N, Knemeyer JP, Sauer M, Wolfrum J (2003) Inter- and intramolecular fluorescence quenching of organic dyes by tryptophan. Bioconjugate Chem 14:1133-1139

Marshall GD, Gaebel T, Matthews JCF, Enderlein J, O'Brien JL, Rabeau JR (2011) Coherence properties of a single dipole emitter in diamond. New J Phys 13:055016

Moerner WE, Fromm DP (2003) Methods of single-molecule fluorescence spectroscopy and microscopy. Rev Sci Instrum 74:3597-3619

Novotny L (2007) Chapter 5 the history of near-field optics. In: Wolf E (ed) Progress in optics, Vol. Volume 50th edn., pp 137-184, Elsevier

Noy A, Huser TR (2003) Combined force and photonic probe microscope with single, molecule sensitivity. Rev Sci Instrum 74:1217-1221

Rothemund PWK (2006) Folding DNA to create nanoscale shapes and patterns. Nature 440:297-302

Rust MJ, Bates M, Zhuang X (2006) Sub-diffraction-limit imaging by stochastic optical reconstruction microscopy (STORM). Nat Meth 3:793-795

Schulz O, Koberling F, Walters D, Koenig M, Viani J, Ros R (2010) Simultaneous single molecule atomic force and fluorescence lifetime imaging. Proc SPIE 7571:757109

Selvin PR (2000) The renaissance of fluorescence resonance energy transfer. NatStructBiol 7:730-734

Steinhauer C, Jungmann R, Sobey TL, Simmel FC, Tinnefeld P (2009) DNA origami as a nanoscopic ruler for super-resolution microscopy. Angew Chem Int Ed Engl 48:8870-8873

van de Linde S, Heilemann M, Sauer M (2012) Live-cell super-resolution imaging with synthetic fluorophores. Annu Rev Phys Chem 63(63):519-540

Westphal V, Kastrup L, Hell SW (2003) Lateral resolution of $28 \mathrm{~nm}$ (lambda/25) in far-field fluorescence microscopy. ApplPhysB 77:377-380

Xie XS, Dunn RC (1994) Probing single-molecule dynamics. Science 265:361-364

Yang TJ, Lessard GA, Quake SR (2000) An apertureless near-field microscope for fluorescence imaging. Appl Phys Lett 76:378-380

Yoskovitz E, Menagen G, Sitt A, Lachman E, Banin U (2010) Nanoscale near-field imaging of excitons in single heterostructured nanorods. Nano Lett 10:30683072

Yoskovitz E, Hadar I, Sitt A, Lieberman I, Banin U (2011) Interplay of quenching and enhancement effects in apertureless near-field fluorescence imaging of single nanoparticles. J Phys Chem C 115:15834-15844

doi:10.1186/2192-2853-2-1

Cite this article as: Schulz et al:: Tip induced fluorescence quenching for nanometer optical and topographical resolution. Optical Nanoscopy 2013 2:1.

\section{Submit your manuscript to a SpringerOpen ${ }^{\odot}$ journal and benefit from:}

- Convenient online submission

- Rigorous peer review

- Immediate publication on acceptance

- Open access: articles freely available online

- High visibility within the field

- Retaining the copyright to your article

Submit your next manuscript at $\gg$ springeropen.com 